\title{
A NOTE ON THE CHARACTERISTIC NUMBERS OF LINEAR SYSTEMS OF DIFFERENTIAL-DIFFERENCE EQUATIONS
}

\author{
JOHN ABRAMOWICH
}

Introduction. The question of stability of solutions of ordinary linear differential equations with variable coefficients is closely related to the determination of their characteristic numbers 1 . Of interest in this connection is the relation between the characteristic numbers of the solutions and the generalized characteristic roots of the system. L. Markus [2] has shown that under certain conditions upper and lower bounds for the characteristic numbers can be determined from the generalized characteristic roots. In this paper we show that a similar result holds for a certain class of linear differentialdifference equations which includes the class treated in [2]. Namely, we establish a relation between the characteristic numbers of the solutions of the system

$$
x_{i}^{\prime}\left(\phi_{i}(t)\right)=\sum_{j=1}^{n} p_{i j}(t) x_{j}\left(\phi_{j}(t)\right) ; \quad i=1, \cdots, n, t \geqq 0,
$$

and the generalized characteristic roots of the determinant

$$
\left|q_{i j}(t)-2 \delta_{i j}\right|=0,
$$

where $q_{i j}(t)=p_{i j}(t) \phi_{i}^{\prime}(t)+p_{j i}(t) \phi_{j}^{\prime}(t)$ and $\delta_{i j}$ is the Kronecker symbol $\left(\delta_{i j}=0, i \neq j\right.$ and $\left.\delta_{i i}=1\right)$.

We recall some pertinent definitions. The real number $\mu$ is said to be the type or Lyapounov number of the real function $f(t)(0 \leqq t<\infty)$ if $\lim \sup _{t \rightarrow \infty}|f(t)| \exp \left(-\mu^{\prime} t\right)=0, \mu^{\prime}>\mu$, and $\lim \sup _{t \rightarrow \infty}|f(t)| \exp \left(-\mu^{\prime \prime} t\right)$ $=\infty, \mu^{\prime \prime}<\mu$. If $|f(t)| \exp (-\mu t)$ is unbounded as $t \rightarrow \infty$ for all positive $\mu$, we say that the type number is equal to $+\infty$, if $|f(t)| \exp (-\mu t)$ approaches zero for all negative $\mu$, we say the type number is equal to $-\infty$. It is easily seen that the type number of the function $f(t)$ is $\lim \sup _{t \rightarrow \infty}(1 / t) \log |f(t)|$. By the characteristic number of the real valued vector function $x(t) \quad(0 \leqq t<\infty)$ we shall mean the largest of the type numbers of its components.

If $a=\left(a_{1}, \cdots, a_{n}\right)$ is any real vector, we shall denote $|a|_{2}=a_{1}^{2}$ $+\cdots+a_{n}^{2}$ and $|a|_{1}=\left|a_{1}\right|+\cdots+\left|a_{n}\right|$.

If $E_{j}=\left\{\phi_{j}(t) \mid \phi_{j}(t) \leqq 0, t \geqq 0\right\}, j=1, \cdots, n$, then $E=U E_{j}$ is called the initial set for the system (1). Assume that $\phi_{j}(t) \rightarrow \infty$, as $t \rightarrow \infty$, $j=1, \cdots, n$, then by a solution of (1) we shall mean any continuous

Received by the editors December 2, 1966. 
vector function $x(t)$ defined on $E \cup[0, \infty)$ and identically satisfying (1) for $t \geqq 0$.

The trivial solution of (1) is said to be asymptotically stable if $\lim _{t \rightarrow \infty}|x(t)|_{1}=0$ for every solution such that $|x(t)|_{1}<\epsilon, t \in E$, for some $\epsilon>0$. Thus the trivial solution of (1) is asymptotically stable if there exists an $\epsilon>0$ such that every solution satisfying for $t \in E$, $|x(t)|_{1}<\epsilon$ has a negative characteristic number. For further details on these questions the reader is referred to the excellent works [3] and $[4]$.

1. We shall make the following assumptions about the system (1):

(a) The functions $p_{i j}(t)$ are continuous and the functions $\phi_{j}(t)$ are continuously differentiable for all $t \geqq 0, i, j=1, \cdots, n$.

(b) The functions $\phi_{j}(t)$ are bounded below and the sets $E_{j}$ $=\left\{\phi_{j}(t) \mid \phi_{j}(t) \leqq 0, t \geqq 0\right\}$ are nonempty, $j=1, \cdots, n$.

(c) The functions $\phi_{j}(t) \rightarrow \infty, t \rightarrow \infty$, and each possesses an inverse monotonically increasing to infinity for $t>t_{1}>0$, which we shall denote by $\phi_{j}^{-1}(t)$.

Under the above conditions the system (1) possesses continuous solutions [3] and the following theorem holds.

ThEOREM. Let $m(t)$ and $M(t)$ be the minimum and maximum roots, respectively, of (2). Then the characteristic numbers of the continuous solutions of the system (1) are contained in the segment $[a, b]$ where

$$
\begin{aligned}
& b=\limsup _{t \rightarrow \infty}\left(\max _{i} \frac{1}{t} \int_{t_{0}}^{\phi_{i}^{-\mathbf{1}}(t)} M(s) d s\right), \\
& a=\limsup _{t \rightarrow \infty}\left(\min _{i} \frac{1}{t} \int_{t_{0}}^{\phi_{i}^{-1}(t)} m(s) d s\right) .
\end{aligned}
$$

Proof. For fixed $t$ let $y_{i}=x_{i}\left(\phi_{i}(t)\right)$ and let $y=\left(y_{1}, \cdots, y_{n}\right)$. Let us find the extreme values of the function

$$
H(t, y)=\sum_{i, j=1}^{n} p_{i j}(t) \phi_{j}^{\prime}(t) y_{i} y_{j}
$$

under the condition $|y|_{2}=1$. Using the method of Lagrange multipliers, we find the extrema of the function $F(t, y)=H(t, y)-\lambda(t)|y|_{2}$. We obtain

$$
0=\frac{\partial F}{\partial y_{k}}=\sum_{j=1}^{n}\left\{p_{j k}(t) \phi_{k}^{\prime}(t)+p_{k j}(t) \phi_{j}^{\prime}(t)\right\} y_{j}-2 \lambda(t) y_{k},
$$

for $k=1, \cdots, n$. Thus the Lagrange multipliers are the roots of (2) 
and multiplying the above equations by $y_{k}$ and adding, we obtain $H(t, y)=\lambda(t)$ and hence $m(t) \leqq H(t, y) \leqq M(t)$. Consequently, for all real $\left(y_{1}, \cdots, y_{n}\right)$ and all $t \geqq 0$, we have the inequalities

$$
m(t)|y|_{2} \leqq H(t, y) \leqq M(t)|y|_{2} .
$$

For the sake of simplicity of notation let us denote the vector function $x_{1}\left(\phi_{1}(t)\right), \cdots, x_{n}\left(\phi_{n}(t)\right)$ by $x(\bar{\phi}(t))$.

Let $x(t)$ be any nontrivial solution of (1) such that $\left|x\left(\bar{\phi}\left(t_{0}\right)\right)\right|_{2}=k^{2}$ for some fixed $t_{0} \geqq 0$. Consider the function

$$
V(t)=|x(\phi(t))|_{2} \exp \left(-\int_{t_{0}}^{t} M(s) d s\right) .
$$

Using the differential equations (1) and the inequality (3), we find $d V / d t \leqq 0$ for all $t \geqq t_{0}$ where $x(\bar{\phi}(t))$ is differentiable. Indeed,

$$
\begin{aligned}
\frac{d V}{d t}= & \frac{d}{d t}|x(\bar{\phi}(t))|_{2} \exp \left(-2 \int_{t_{0}}^{t} M(s) d s\right) \\
& -2 M(t)|x(\bar{\phi}(t))|_{2} \exp \left(-2 \int_{t_{0}}^{t} M(s) d s\right) .
\end{aligned}
$$

Using the system (1) we find that $(d / d t)|x(\bar{\phi}(t))|_{2}=2 H(t, x(\bar{\phi}(t)))$, except possibly at those values of $t$ where the left-hand side does not exist, and the result follows from (3). Since $x(\Phi(t))$ is continuous, we have for all $t \geqq t_{0}$

$$
|x(\bar{\phi}(t))|_{2} \leqq k^{2} \exp \left(2 \int_{t_{0}}^{t} M(s) d s\right) .
$$

In a similar way, using the function

$$
V(t)=|x(\bar{\phi}(t))|_{2} \exp \left(-2 \int_{t_{0}}^{t} m(s) d s\right)
$$

we obtain

$$
|x(\bar{\phi}(t))|_{2} \geqq k^{2} \exp \left(2 \int_{t_{0}}^{t} m(s) d s\right) .
$$

Therefore, for all $t \geqq t_{0}$,

(4) $k^{2} \exp \left(2 \int_{t_{0}}^{t} m(s) d s\right) \leqq|x(\bar{\phi}(t))|_{2} \leqq k^{2} \exp \left(2 \int_{t_{0}}^{t} M(s) d s\right)$.

Since $x_{i}^{2}\left(\phi_{i}(t)\right) \leqq k_{1}^{2} \exp \left(2 \int_{t_{0}}^{t} M(s) d s\right)$ implies 
$x_{i}^{2}(t) \leqq k_{2}^{2} \exp \left(2 \int_{t}^{\phi_{i}^{-1}(t)} M(s) d s\right) \leqq k_{2}^{2} \exp \left(\max _{t} 2 \int_{t}^{\phi_{i}^{-1}(t)} M(s) d s\right)$,

we have

$$
|\bar{x}(t)|_{2} \leqq k^{\prime} \exp \left(\max _{i} 2 \int_{t_{\theta}}^{\phi_{i}^{-1}(t)} M(s) d s\right)
$$

for some positive constant $k^{\prime}$ and by assumption (c) for all $t \geqq t_{0}$. In a similar way, we show that

$$
|x(t)|_{2} \geqq k^{\prime \prime} \exp \left(\min 2 \int_{t_{0}}^{\phi_{i}^{-1}(t)} m(s) d s\right)
$$

for some positive constant $k^{\prime \prime}$ and $t \geqq t_{0}$. Thus

$$
\begin{aligned}
k^{\prime \prime} \exp \left(\min _{i} \int_{t}^{\phi_{i}^{-1}(t)} m(s) d s\right) & \leqq|x(t)|_{2} \\
& \leqq k^{\prime} \exp \left(\max _{i} 2 \int_{t_{0}}^{\phi_{i}^{-1}(t)} M(s) d s\right) .
\end{aligned}
$$

Since the type number of $f^{2}(t)$ is twice that of $f(t)$, the characteristic number of $x(t)$ is equal to half the type number of $|x(t)|_{2}$ and the theorem follows from the above inequalities.

Corollary 1. If

$$
\lim _{t \rightarrow \infty} \frac{1}{t}\left(\max _{i} \int_{t_{0}}^{\phi_{i}^{-1}(t)} M(s) d s\right)=-c, \quad c>0,
$$

then the trivial solution of (1) is asymptotically stable.

Corollary 2. If

$$
\limsup _{t \rightarrow \infty} \frac{1}{t}\left(\min _{i} \int_{t_{0}}^{\phi_{i}^{-1}(t)} m(s) d s\right)=c, \quad c>0,
$$

then the trivial solution of (1) is unstable.

Remark. Assumption (c) was required to insure that the inequalities (4) and (5) hold for all $t \geqq t_{0}$. These could hold on some finite interval without this assumption.

As an example let us take the second order system

$$
\begin{aligned}
x^{\prime}(t) & =\left(\sin t-\cos ^{2} t\right) x(t)+p_{12}(t) y\left(\frac{1}{2} t\right), \\
y^{\prime}\left(\frac{1}{2} t\right) & =p_{21}(t) x(t)-4\left(\sin t+2 \cos ^{2} t\right) y\left(\frac{1}{2} t\right),
\end{aligned}
$$


where $p_{12}(t)+\frac{1}{2} p_{21}(t)=0$.

We find $M(t)=\sin t-\cos ^{2} t$ and

$$
\limsup _{t \rightarrow \infty} \frac{1}{t} \int_{0}^{2 t}\left(\sin s-\cos ^{2} s\right) d s=-1
$$

and therefore by Corollary 1 the trivial solution is asymptotically stable.

\section{REFERENCES}

1. A. M. Lyapounov, Problème générale de la stabilité du mouvement, Princeton Univ. Press, Princeton, N. J., 1947.

2. L. Markus, Continuous matrices and the stability of differential systems, Math. Z. 62 (1955), 310-319.

3. L. E. Elsgolts, Introduction to the theory of differential equations with retarded arguments, Moscow, 1964. (Russian)

4. R. Bellman and K. L. Cooke, Differential-difference equations, Academic Press, New York, 1963.

Wayne State University 